\title{
ON PROGRESS AND METHODS OF REDUCTION OF THE MELBOURNE OBSERVATIONS OF FUNDAMENTAL STARS
}

\author{
K. N. TAVASTSHERNA \\ Pulkovo Observatory, U.S.S.R.
}

The astronomers C. Merfield, J. Moroney, G. Wootthouse, J. Feely and W. Holmes of the Melbourne Observatory made a large series of observations of fundamental stars with the 8" meridian circle (Troughton and Simms) during 1928-1941. After closing the Observatory all the materials concerned with this large work (about 20000 observations in $\delta$ and 30000 observations in $\alpha$ ) were forwarded to the Mount Stromlo and then the Pulkovo Observatories for the final reduction and publication (Tavastsherna, 1963).

The reduction of declinations has now been completed. Four absolute catalogues have been published (Tavastsherna and Shakht, 1968). These catalogues include: (1) 2249 Backlund-Hough list stars from $+32^{\circ}$ to $-90^{\circ}$ of declinations $\left(\mathrm{Me}_{50} \mathrm{I} \delta\right),(2) 144$ latitude stars in the Kimura list $\left(\mathrm{Me}_{50} \mathrm{II} \delta\right)$, (3) 35 stars with the $+32^{\circ}--90^{\circ}$ declinations from the A. Kopff list of 'Ersatzsternen' ( $\mathrm{Me}_{50} \mathrm{III} \delta$ ) and (4) 38 zenith stars of the Melbourne Observatory $\left(\mathrm{Me}_{50} \mathrm{IV} \delta\right.$ ).

At present the first stage of the reduction of right ascensions of these stars has also been completed. The differential catalogue was compiled in the $\mathrm{N} 30$ system. We propose to compile an absolute catalogue using a combination of the step-by-step, chain and Greenwich methods.

\section{References}

Tavastsherna, K. N.: 1963, Trudy 15th Astrometr. Konf., p. 156.

Tavastsherna, K. N. and Shakht, N. A.: 1968, Catalogues of Stellar Positions Compiled at Pulkovo Observatory on the Basis of Observations made at Melbourne Observatory from 1928 to 1941, Pulkovo, Leningrad. 